\title{
Magneto-optical spin Hall effect of light controlled by thermo-optical effect
}

\author{
Tingting Tang ${ }^{1, *}$, Pengyu Zhang ${ }^{1}$,Yuanxun $\mathrm{Li}^{2}$, Chaoyang $\mathrm{Li}^{3}$, Xiaolei Huang ${ }^{4}$, Yang $\mathrm{Cao}^{4}$, \\ Ming $\mathrm{Wan}^{4}$, Fei Xiong ${ }^{5}$ and Haijun $\mathrm{Yao}^{6}$ \\ ${ }^{1}$ Information Materials and Device Applications Key Laboratory of Sichuan Provincial Universities, \\ Chengdu University of Information Technology, Chengdu, Sichuan, 610225, China \\ ${ }^{2}$ State Key Laboratory of Electronic Thin Films and Integrated Devices, University of Electronic \\ Science and Technology of China, Chengdu, Sichuan, 610054, China \\ ${ }^{3}$ State Key Laboratory of Marine Resource Utilization in South China Sea, Hainan University, No. 58, \\ Renmin Avenue, Haikou, Hainan, 570228, China \\ ${ }^{4}$ Eoptolink Technology Inc., Ltd, No.127 West Wulian Street, Shuangliu District, Chengdu, 610213, \\ China \\ ${ }^{5}$ Shennzhen Huayang Technology Development Co.,Ltd., No. 1 Shaodi Road, Nanshan District, \\ Shenzhen, Guangdong, 518000, China \\ ${ }^{6}$ Shennzhen Apat Opto-Electronics Components Co.,Ltd., Chengdu R\&D Center, Chengdu, Sichuan, \\ 610000, China
}

\begin{abstract}
In this paper, an experimental method of magneto-optical spin Hall effect of light (MOSHEL) based on thermo-optical control is designed. First, we design and implement a pilot experiment - the SHEL experiment controlled by thermo-optical. According to the experiment, it is concluded that the increase of the temperature of the thermo-optical medium will have an important influence on the corresponding thermo-optical spin Hall effect of light (TOSHEL). Accordingly, we designed a device that controls the SHEL in magneto-optical-thermo-optical (MO-TO) two-dimension that uses the thermo-optical effect to simultaneously control the intrinsic TOSHEL and MOSHEL.
\end{abstract}

\section{Introduction}

In 2004, Onoda of AIST, Japan, for the first time, clearly proposed the photonic spin Hall effect: when a beam of linearly polarized light propagates in a non-uniform medium, photons with opposite spins move along the direction perpendicular to the refractive index gradient ${ }^{[1]}$. Eventually it will cause the outgoing beam to split into two circularly polarized beams and separate on both sides of the outgoing beam section. Since the spin Hall effect of light (SHEL) was proposed, it has received extensive attention from researchers. In recent years, people have achieved remarkable results in the enhancement ${ }^{[2-5]}$, control ${ }^{[6-10]}$, and other aspects of SHEL ${ }^{[1-14]}$. At present, some scholars have designed refractive index sensors ${ }^{[15-17]}$ and biosensors ${ }^{[18]}$ based on SHEL.

\footnotetext{
*Corresponding author: skottt@163.com
} 
MOSHEL ${ }^{[19-21]}$ as a novel method of regulating photon spin has received more and more attention. According to the different magnetization directions, the MOSHEL can be divided into three situations: LMOKE, PMOKE, and TMOKE ${ }^{[22]}$. Longitudinal magneto-optical spin Hall effect, the magnetization direction is parallel to the incident surface and the surface of the magneto-optical medium at the same time, the pole magneto-optical spin Hall effect, the magnetization direction is parallel to the incident surface and perpendicular to the surface of the magneto-optical medium. Transverse magneto-optical optical spin Hall effect, the magneto-optical direction is perpendicular to the incident surface and the surface of the magneto-optical medium at the same time.

As a source of magnetic field, permanent magnets have become the first choice of many researchers because of their low cost, small size, flexible operation, customizable size, and high magnetic field strength. The other side of the coin is the use of permanent magnets as a magnetic field source has its inherent limitations: once a permanent magnet is manufactured, its magnetic field strength cannot be adjusted, which limits the study of MOSHEL. If an electromagnet is used as a magnetic field source, its magnetic field strength can be effectively controlled by adjusting the magnitude of the input current, and even a multi-dimensional adjustment of the direction of the magnetic field can be achieved through a precisely designed electromagnet. However, if the above functions are to be achieved and the magnetic field strength required by the experimental design is achieved, the volume of the electromagnet will be very large and heavy, and its cost is extremely high, which is tens of thousands of times the cost of using a permanent magnet as a magnetic field source, and existing resources cannot meet the requirements. Therefore, we hope to effectively regulate the MOSHEL in a new way - the thermo-optical effect.

\section{Theory and experiment results of TOSHEL}

For incident light with a finite width, its actual wave surface is in the form of a wave packet, so it can be regarded as a superposition of multiple plane waves, which have different angular spectrum components. When the medium is reflected, it will produce different additional phases and interfere with each other. The final macroscopic performance is the redistribution of the optical field energy. Therefore, the angular spectrum theory can be used to perform analytical calculations on SHEL. Angular spectrum theory is to transform the incident light field into an angular spectrum form, and then use the relationship between reflection and incident angle spectrum to calculate the exit angle spectrum, and then obtain the light field expression of the exit angle spectrum, and finally use the mathematical integration method obtains the geometric position of the center of mass of the light field.

The thermo-optical effect refers to the change in the molecular arrangement of the dielectric by heating or cooling, which causes the optical properties of the dielectric to change with temperature. Normally, the change in optical properties is mainly reflected in the change in the refractive index or dielectric constant of the medium as the temperature of the medium changes. When a beam of linearly polarized light propagates in a non-uniform medium, the propagation direction of the light changes, and the SHEL will occur. As the medium changes in temperature, the refractive index changes, and the SHEL also changes. As a thermo-optical phase change material, $\mathrm{VO}_{2}$ will change from a semiconductor state at a low temperature to a metal state at a high temperature when heated to $68{ }^{\circ} \mathrm{C}$. At this time, its dielectric constant will undergo a sudden change from positive to negative. In addition, thermo-optical materials usually have a reverse change process in their optical properties when the temperature drops.

Therefore, we speculate that SHEL of $\mathrm{VO}_{2}$ reflected light will change drastically during the heating process, and the change law of the SHEL after cooling is the inverse process of heating. In order to verify our speculation, we designed and fabricated a $\mathrm{VO}_{2}$ sample for 
TOSHEL experiment. Its structure is shown in Fig.1. In order to achieve controllable heating of the entire $\mathrm{VO}_{2}$ sample, we used a silicone rubber heater as an external heat source.

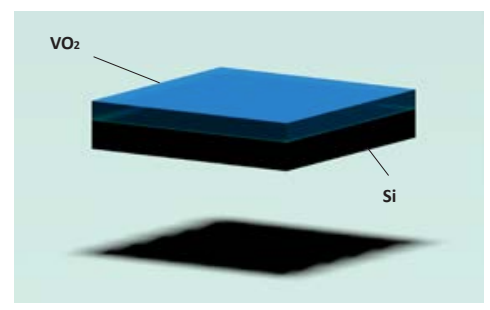

Fig. 1. The sample structure diagram of $\mathrm{VO}_{2}$.

By observing and recording the change of the light field centroid received by the CCD at different setting temperatures $\mathrm{T}$, value of the spin transverse shift of the TOSHEL as shown in Fig. 2.

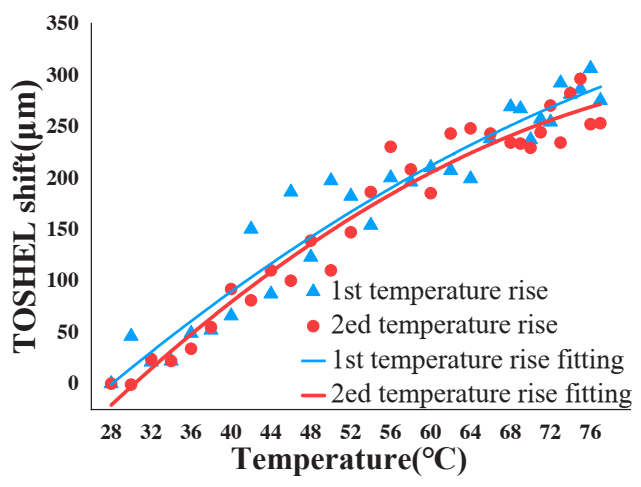

Fig. 2. The change of TOSHEL shift with temperature when heating up.

The triangle points and circle points in Fig. 2 are the changes of TOSHEL shift with the increase of temperature in the first and second experiments, respectively. The single solid line and double solid line are the polynomial fitting curves of the above-mentioned points. It can be seen from Fig. 2 that the TOSHEL shift increases with the increase of sample temperature. The fitting curves of the two experiments are convex, which indicates that the growth rate decreases slowly with the increase of temperature, and its instability also decreases gradually with increasing temperature. The TOSHEL shift is about 250 microns at $76^{\circ} \mathrm{C}$, showing strong TOSHEL.

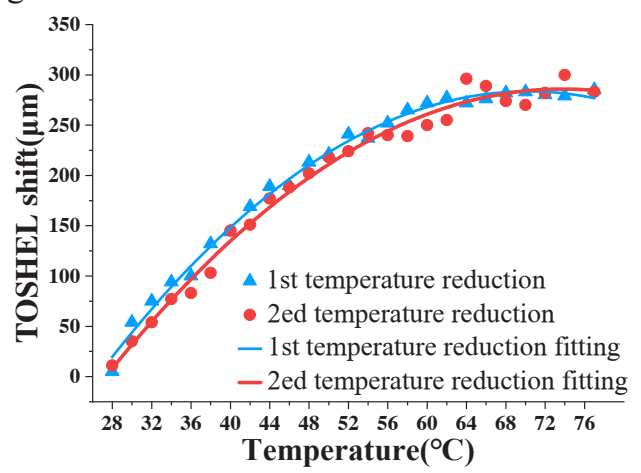

Fig. 3. The change of TOSHEL shift with temperature during cooling. 
The triangle point and the circle point in Fig. 3 are the changes of TOSHEL shift with the decrease of temperature in the first and second experiments, respectively. The single solid line and the double solid line are the quadratic fitting curves of the above-mentioned point sequence. For the convenience of comparison with the heating experiment, the data of the cooling experiment is drawn from right to left. It can be seen from Fig. 3 that TOSHEL shift increases with the increase of temperature, reaching an extreme value of about $250 \mu$ $\mathrm{m}$ at $76^{\circ} \mathrm{C}$. Different from Figure 2, the change of TOSHEL shift is relatively smooth in the cooling process, and the oscillation in the high temperature region is slightly greater than that in the low temperature region.

Comparing Fig. 2 and Fig. 3, it can be found that the fitting curve trend of the same type of experiment is consistent, indicating that the thermo-optical spin Hall experiment has good repeatability. The fitting curves of the first experiment (including the first heating experiment and the first cooling experiment) and the second experiment are also very close, indicating that the thermo-optical spin Hall effect experiment has good temperature reversibility.

\section{Conclusion}

In this paper, a magneto-optical spin Hall effect based on thermo-optical control is designed. To achieve this goal, we designed and produced $\mathrm{VO}_{2}$ samples. The heating equipment and temperature control device of the thermo-optical material are determined, and the effective control of the temperature of the thermo-optical material is achieved. Then designed TOSHEL's experimental light path to prepare material for the successful implementation of the experiment. Finally, we proposed a SHEL based on MO-TO two-dimensional control and design an experimental optical path. Through analysis, it is concluded that the introduction of thermo-optical effect will not only produce intrinsic TOSHEL shift, but also the change in refractive index of the medium produced by thermo-optical effect will also cause drastic changes in MOSHEL. This pioneering design opens up new ideas and directions for the flexible regulation of MOSHEL and its sensing applications.

This work is supported by Sichuan Science and Technology Program (2019JDJQ0003 and 2020YFQ0040).

\section{References}

1. M. Onoda, S. Murakami, and N. Nagaosa, Hall effect of light[J], Phys. Rev. Lett. 93,8: 083901(2004)

2. A. P. Slobozhanyuk, A. N. Poddubny, I. S. Sinev, et al, Enhanced photonic spin Hall effect with subwavelength topological edge states[J]. Laser \& Photonics Reviews, 10,4:656-664(2016)

3. H. Luo, X. Ling, X. Zhou, et al, Enhancing or suppressing the spin Hall effect of light in layered nanostructures[J]. Physical Review A, 84,3:033801(2011)

4. H. Luo, X. Zhou, W. Shu, et al. Enhanced and switchable spin Hall effect of light near the Brewster angle on reflection[J]. Physical Review A, 84,4:043806(2011)

5. Tingting. T, Jie. L, Li. L, et al. Loss enhanced spin Hall effect of transmitted light through anisotropic epsilon- and mu-near-zero metamaterial slab[J]. Optics Express, 25,3:2347-(2017)

6. X. Ling, X. Zhou, K. Huang, et al. Recent advances in the spin Hall effect of light[J]. Reports on Progress in Physics, 80,6:066401(2017) 
7. L. Luo, G. Feng, S. Zhou, et al. Photonic spin Hall effect of reflected light in a prismgraphene waveguide[J]. Superlattices \& Microstructures, 122(OCT.):530-537(2018)

8. X. Yin, Z. Ye, J. Rho, et al. Photonic Spin Hall Effect at Metasurfaces[J]. Science, 339,6126:1405-1407(2013)

9. Z. Li, W. Liu, H. Cheng, et al. Manipulation of the Photonic Spin Hall Effect with High Efficiency in Gold-Nanorod-Based Metasurfaces[J]. Advanced Optical Materials, 5,20:1700413(2017)

10. Y. U. Lee, J. W. Wu. Control of optical spin Hall shift in phase-discontinuity metasurface by weak value measurement post-selection[J]. Scientific Reports, 5:13900(2015)

11. X. Zhou, Z. Xiao, H. Luo, et al. Experimental observation of the spin Hall effect of light on a nanometal film via weak measurements[J]. Physical Review A, 85,4:15(2012)

12. K. Y. Bliokh, Y. P. Bliokh, Conservation of angular momentum, transverse shift, and spin Hall effect in reflection and refraction of an electromagnetic wave packet[J], Phys. Rev. Lett, 96,7: 073903(2006)

13. X. Zhou, X. Ling, Z. Zhang, et al. Observation of Spin Hall Effect in Photon Tunneling via Weak Measurements[J]. Scientific Reports, 4: 7388(2014)

14. Xinxing. Zhou, Xing. Li, Hailu. Luo, et al. Optimal preselection and postselection in weak measurements for observing photonic spin Hall effect[J]. Applied Physics Letters, 104,5:051130(2014)

15. Sheng. L, Xie. L, H. Luo, et al. Sensitivity Enhanced Refractive Index Sensor by Reducing the Influence of In-Plane Wavevector in Photonic Spin Hall Effect[J]. IEEE Photonics Journal, 10,5:1-9(2018)

16. X. Zhou, L. Sheng, X. Ling. Photonic spin Hall effect enabled refractive index sensor using weak measurements[J]. Scientific Reports, 8,1:1221(2018)

17. P. Zhang, T. Tang, L. Luo, et al. Magneto-optical spin Hall effect of light and its application in refractive index detection[J]. Optics Communications,:126175(2020).

18. N. Li, T. Tang, J. Li, et al. Highly sensitive biosensor with Graphene-MoS2 heterostructure based on photonic spin Hall effect[J]. Journal of Magnetism and Magnetic Materials, 484(AUG.):445-450(2019)

19. Tingting. Tang, Jie. Li, Li. Luo, et al. Magneto-Optical Modulation of Photonic Spin Hall Effect of Graphene in Terahertz Region[J]. Advanced Optical Materials, 6,7:1701212.1-1701212.7(2018)

20. J. Li, T. Tang, L. Luo, et al. Weak measurement of the magneto-optical spin Hall effect of light[J]. Photonics Research, 7,9: 1014(2019)

21. X. Qiu, X. Zhou, D. Hu, et al. Determination of magneto-optical constant of Fe films with weak measurements[J]. Applied Physics Letters,105,13:131111.1-131111.4(2014)

22. G.Armelles,A.Cebollada, A. García-Martín, and M. U. González, "Magnetoplasmonics: combining magnetic and plasmonic functionalities," Adv. Opt. Mater. 1, 10-35 (2013). 